\title{
Tackling down the low wind effect on SPHERE instrument
}

Jean-François Sauvage, Thierry Fusco, Masen Lamb, Julien Girard, Martin Brinkmann, et al.

Jean-François Sauvage, Thierry Fusco, Masen Lamb, Julien Girard, Martin Brinkmann, Andres Guesalaga, Peter Wizinowich, Jared O'Neal, Mamadou N'Diaye, Arthur Vigan, David Mouillet, Jean-Luc Beuzit, Markus Kasper, Miska Le Louarn, Julien Milli, Kjetil Dohlen, Benoît Neichel, Pierre Bourget, Pierre Haguenauer, Dimitri Mawet, "Tackling down the low wind effect on SPHERE instrument," Proc. SPIE 9909, Adaptive Optics Systems V, 990916 (26 July 2016); doi: 10.1117/12.2232459

Event: SPIE Astronomical Telescopes + Instrumentation, 2016, Edinburgh, United Kingdom 


\title{
Tackling down the Low Wind Effect on SPHERE instrument
}

\author{
Sauvage Jean-François, ${ }^{a, b}$ Fusco Thierry, ${ }^{a, b}$ Lamb Masen ${ }^{\mathrm{j}}$, Girard Julien ${ }^{\mathrm{f}}$, Brinkmann Martin ${ }^{\mathrm{f}}$, \\ Guesalaga Andres, ${ }^{\text {, b, d }}$ Wizinowich Peter, ${ }^{\mathrm{e}}$ O'Neal Jared, ${ }^{\mathrm{f}} \mathrm{N}^{\prime}$ Diaye Mamadou, ${ }^{\mathrm{g}}$ Vigan Arthur, ${ }^{\mathrm{b}}$ \\ Mouillet David, ${ }^{\mathrm{h}}$ Beuzit Jean-Luc, ${ }_{\mathrm{h}}$ Kasper Markus, ${ }^{\mathrm{f}}$ Le Louarn Miska, ${ }^{\mathrm{f}}$ Milli Julien, ${ }^{\mathrm{f}}$ Dohlen Kjetil, ${ }^{\mathrm{b}}$ \\ Neichel Benoît, ${ }^{\mathrm{b}}$ Bourget Pierre, ${ }^{\mathrm{f}}$ Haguenauer Pierre, ${ }^{\mathrm{f}}$ Mawet Dimitri ${ }^{\mathrm{i}}$ \\ ${ }^{a}$ ONERA - Optics Department, 29 avenue de la Division Leclerc, F-92322 Chatillon Cedex, France \\ ${ }^{\mathrm{b}}$ Aix Marseille Universite, CNRS, LAM (Laboratoire d'Astrophysique de Marseille) UMR 7326, 13388, Marseille, \\ France \\ ${ }^{\mathrm{c}}$ Department of Electrical Engineering, School of Engineering, Pontificia Universidad Católica de Chile \\ ${ }^{\mathrm{d}}$ Fondation IMéRA (L'institut d'études avancées d'Aix-Marseille) 2, Place Le Verrier 13004 Marseille-FranceIMERA, \\ ${ }^{\mathrm{e}}$ W. M. Keck Observatory, 65-1120 Mamalahoa Hwy, Kamuela, HI, USA 96743 \\ ${ }^{\mathrm{f}}$ ESO European Southern Observatory, Karl-Schwarzschild-Strasse 2, D-85748 Garching \\ ${ }^{\mathrm{g}} \mathrm{STScI}$ Space Telescope Science Institute, Baltimore \\ ${ }^{\text {h }}$ IPAG UJF-Grenoble 1 / CNRS-INSU, Institut de Planétologie et d'Astrophysique de Grenoble (IPAG) UMR 5274, \\ Grenoble, F-38041, France \\ i JPL, Pasadena, USA \\ ${ }^{\mathrm{j}}$ University of Victoria, 3800 Finnerty Rd, Victoria, Canada and NRC Herzberg Astronomy, 5071 W. Saanich Rd, \\ Victoria, Canada
}

\begin{abstract}
SPHERE is the VLT second generation planet hunter instrument. Installed since may 2014 on UT3, the system has been commissionned and verified for more than one year now and routinely delivers unprecedented images of star surroundings, exoplanets and dust disks. The exceptionnal performance required for this kind of observation makes the appointment: a repeatable Strehl Ratio of $90 \%$ in $\mathrm{H}$ band, a rough contrast level of 10-5@0.5 arcsec, and reaches 10-6 at the same separation after differential imaging (SDI, ADI). The instrument also presents high contrast levels in the visible and an unprecedented 17mas diffraction-limited resolution at 0.65 microns wavelength. SAXO is the SPHERE XAO system, allowing the system to reach its final detectivity. Its high performance and therefore highly sensitive capacities turns a new eye on telescope environement. Even if XAO performance are reached as expected, some unexpected limitations are here described and a first work around is proposed and discussed. Spatial limitation: wave-front aberrations have been identified, deviating from kolmogorov statistics, and therefore not easily seen and compensated for by the XAO system. The impact of this limitations results in a degraded performance in some particular low wind conditions. Solutions are developped and tested on sky to propose a new operation procedure reducing this limitation. Temporal limitation: high amplitude vibrations on the low order modes have been issued, due to telescope environment and XAO behaviour. Again, a solution is developped and an assessment of its performance is dressed. The potential application of these solutions to E-ELT is proposed.
\end{abstract}

Keywords: High contrast imaging, adaptive optics, dome seeing

Adaptive Optics Systems V, edited by Enrico Marchetti, Laird M. Close, Jean-Pierre Véran, Proc. of SPIE Vol. 9909,990916 (c) 2016 SPIE · CCC code: 0277-786X/16/\$18 · doi: 10.1117/12.2232459 


\section{INTRODUCTION}

The direct imaging of extra-solar planets is one of the most exciting challenges of today's astronomy. From a scientific point-of-view, the light coming from an extra-solar planet is able to reveal the chemical elements of its atmosphere. Direct imaging of exoplanets therefore enables in a short term the search for habitable planets and eventually extraterrestrial life. The year 2014 has seen a real revolution with the delivery of an instruments fully dedicated to high contrast, and opened to a wide community: SPHERE (Spectro Polarimetric High contrast Exoplanet REsearch) is one of them. It already began to provide lots of science.

As explained in [1], a strong limitation called Low Wind Effect [LWE] to the SPHERE instrument detectivity capacity has been identified during the instrument commissioning in 2014. In the previous paper, the limitation has been identified as a strong phase aberrations of typical shape shown in Figure 1 [a for global pupil measurement] [b for close-up view on a spider] [c for the effect as seen on DTTS imager]. Some very sharp discontinuities in phase aberrations located around the spiders and of very large amplitudes up to hundreds of nanometers have been measured during onsky measurement on bright star.

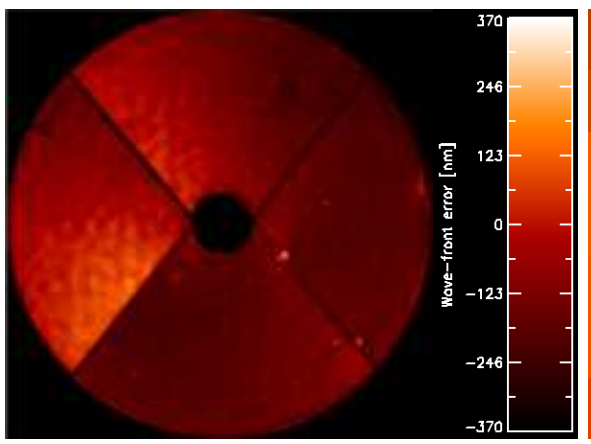

(a)

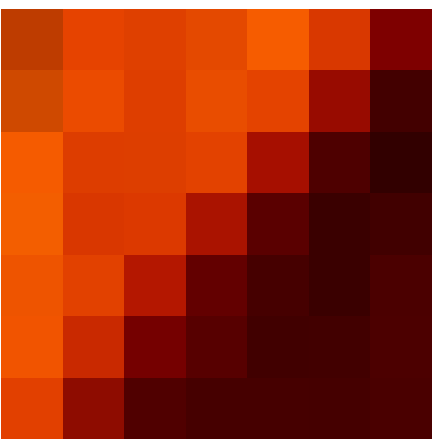

(b)

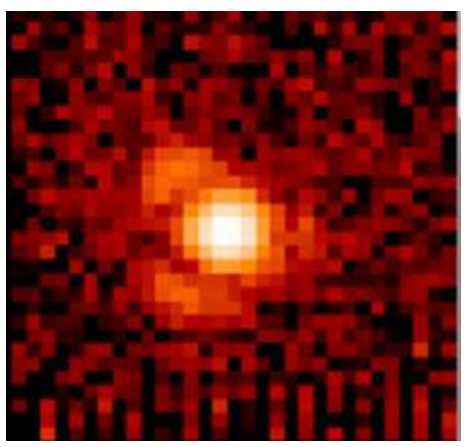

(c)

Figure 1: Wave-front aberration measured by ZELDA WFS of SPHERE, during on-sky measurement of commissioning 4. (a) Measurement taken on the whole pupil. (b) Zoom of (a) on an area covered by one subaperture, along the spider arm bottom/left. The aberration presents strong discontinuities up to hundreds of nanometers (optical wave-front). (c) effect of LWE pattern in the focal plane of DTTS, $H$ band imaging.

Of course the (c) image in Figure 1 clearly illustrates how difficult it is to perform high contrast imaging with such a defect.

\section{SCENARIO OF LWE}

The scenario that we propose to explain the LWE pattern is based on radiative transfer between the spider and the sky background, decreasing the temperature of the spider by a large amount. The air coming on the spider has therefore a temperature higher than the spider (see Figure 2), and therefore loses heat when crossing the spider. The air after the spider has a decreased temperature T' ${ }_{\text {air }}$. This temperature difference creates a temperature gradient of the air on each side of the spider arm. As an example, a temperature difference of $1^{\circ}$, cumulated on a $1 \mathrm{~m}$ height, is responsible for an optical path difference of $800 \mathrm{~nm}$ between each side of the spider.

This scenario has the advantage to explain the following points: 
- Such a scenario explains that the optical path difference profile presents a sharp step, the temperature varies very rapidly across the spider

- The OPD created by the air difference will increase when the wind speed decreases.

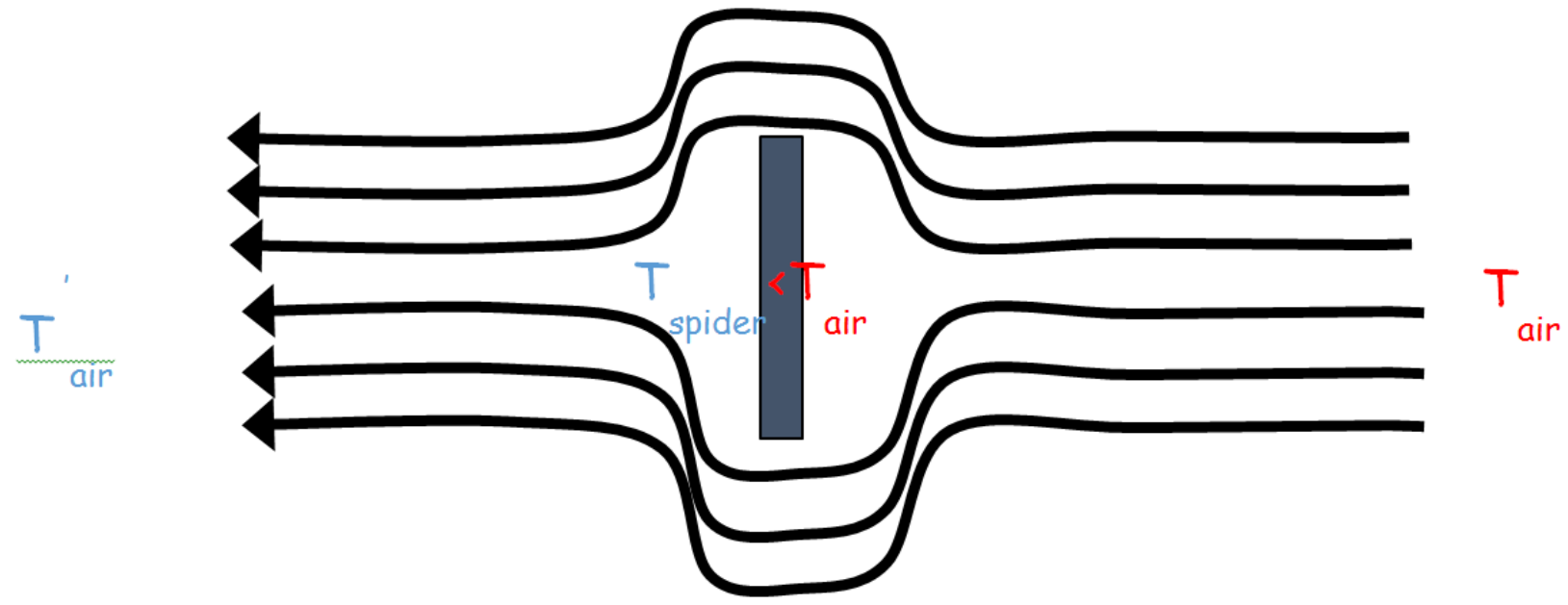

Figure 2: Scenario of the LWE : the radiative transfer decreases the temperature of the spider. Some heat exchange exist between the air at temperature $T_{\text {air }}$ and the spider, leading to a difference

\section{AIR FLOW SIMULATIONS}

ESO has driven a simulation analysis of the air flow around the spiders. The CFD simulations have been driven with an ANSYS software, based on a 2D simplified geometry. The spider is assumed to be a vertical body, colder than the incoming air flow by $3 \mathrm{~K}$. The Figure 3 shows the results of the numerical simulations. The 2D maps of temperature are shown in a cross section of the spider, the air flow is coming from the left. At high wind velocity $(3 \mathrm{~m} / \mathrm{s}$, left), the air temperature after the spider decreases by $0.1 \mathrm{~K}$. At a lower velocity $(0.3 \mathrm{~m} / \mathrm{s}$, right), the air temperature after the spider is much colder, down to $1 \mathrm{~K}$ difference just after the spider. The heat exchange process is made more efficient due to the lower wind speed. The Optical Path Difference created by such a temperature difference might reach $100 \mathrm{~nm}$, when cumulated on a $20 \mathrm{~cm}$ height profile.

These preliminary results are fully confirming the scenario proposed in [1]. The next step of this work is to go for a 3D modelisation of the air flow inside Unit Telescope. Another step is to transform these temperature maps into OPD maps, in order to compare these maps with the measurements made on SPHERE. 

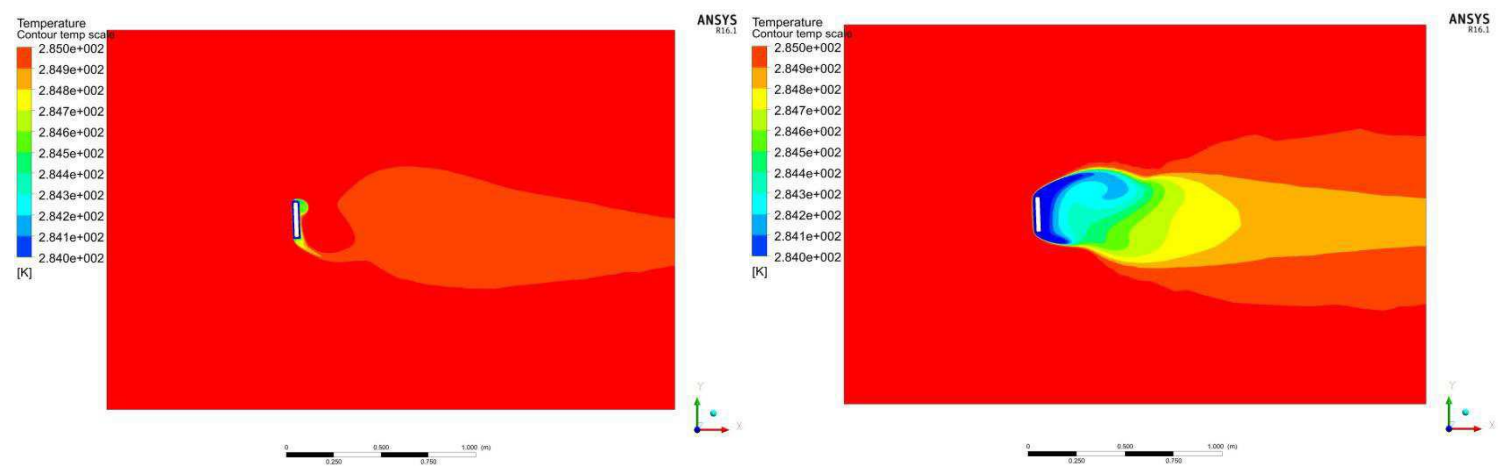

Figure 3: ANSYS simulations results. The 2D temperature map in a vertical plane orthogonal to the spider direction is shown. Air flow is coming from the left, telescope is at the bottom.

\section{LWE CONDITION OF APPARITION}

\subsection{Spider profile}

The LWE is depending on the spider geometry and profile. There are as many spider profiles as existing telescopes over the world. In Figure 4 some profiles are shown for Keck, VLT-UT and Subaru telescope. The spider profile of Keck is known to be efficient to avoid LWE: thin tubes spiders offering a small cross-section to the wind, and a painting slowing down the radiative transfert process. VLT-UT has demonstrated a strong and regular LWE process, due to the spider crosssection ( 2 sections of $20-30 \mathrm{~cm}$ height). According to the previous analysis and scenario, the Subaru spider profile seems to be very efficient at producing LWE, due to the plain and large spiders, black painted. Exchanges with Subaru SceXao tem confirmed that they are quite often suffering from this effect.

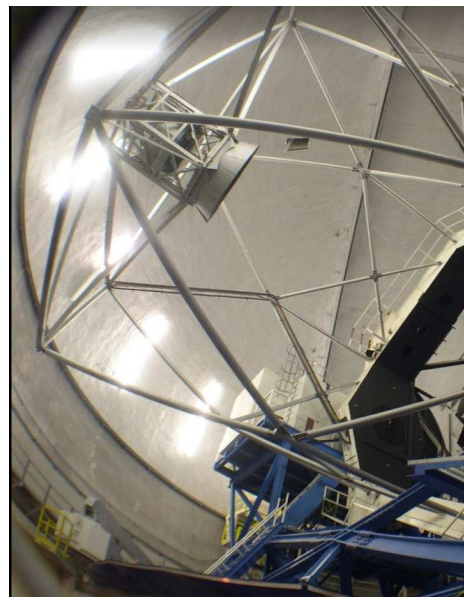

Keck

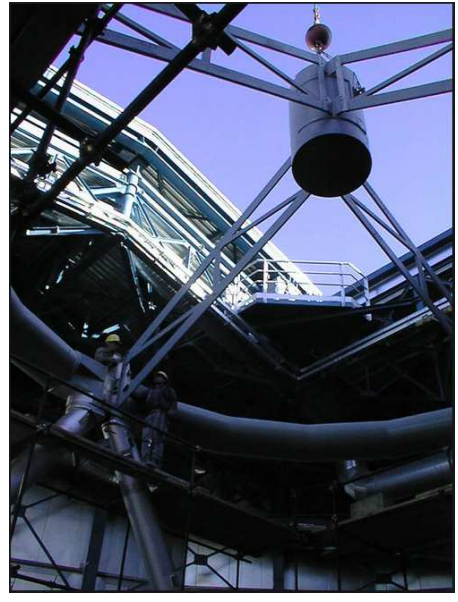

VLT UT

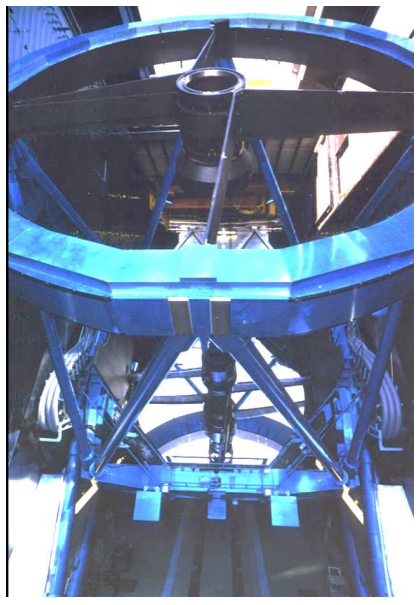

Subaru

Figure 4: 3 examples of telescope spider.

\subsection{Wind statistics}

The wind speed at the level of the telescope top ring seems to be a key parameter to detect or predict the apparition of LWE. Wind speeds measured at this level below $1 \mathrm{~m} / \mathrm{s}$ drives the system in a regime 
out of high contrast capacities. Measuring small wind speed is not always an easy task, and a systematic study of the apparition of LWE on SPHERE has shown that the phenomen appears as soon as the wind speed measured at $30 \mathrm{~m}$ height drops below $3 \mathrm{~m} / \mathrm{s}$. The fraction of nights below $3 \mathrm{~m} / \mathrm{s}$ at Paranal is estimated at 20\% in the last 17 years Figure 5, hence confirming the fraction of night with LWE seen on SPHERE.

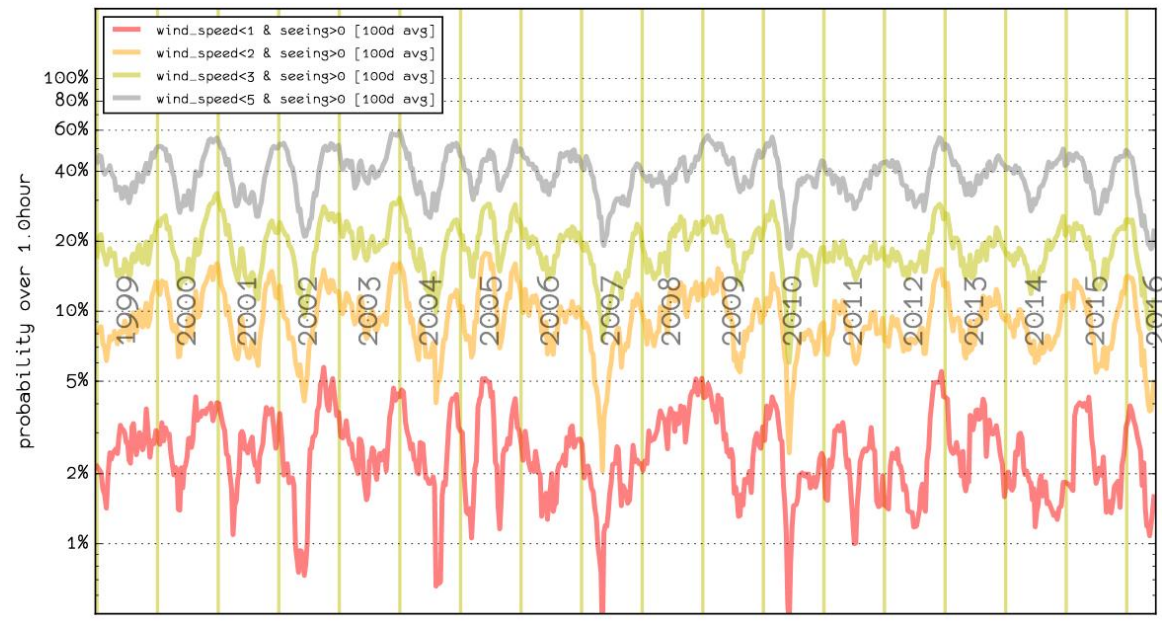

Figure 5 : wind statistics as measured at 30m height at Paranal since 1999.

\section{HOW TO MEASURE LWE}

The LWE typical aberration pattern is hard to measure, because it consists of sharp and strong phase jumps in the pupil. An exhaustive study has been driven together by ONERA, LAM and University of Victoria on the measurement of LWE pattern in SPHERE instrument. There are several ways to perform WFS on SPHERE. These ways are listed here, with a first discussion about their relevance to measure the LWE pattern, both in term of performance and operation.

- Zernike Wave-Front Sensor is able to measure phase aberrations with extremely high spatial resolution. This WFS has demonstrated its ability to measure the LWE by itself, as it was able to first diagnose the problem. The bad point with this WFS on SPHERE is that it is not quick to set. As the LWE can evolve at a characteristic time down to 1 second, the WFS has to be done quicker or at least at this frame rate.

- Shack-Hartmann WFS of SPHERE is a 40x40 spatially filtered SH. It is apparently not well suited to measure this effect, as the Center Of Gravity measurement is not directly sensitive to a differential piston step inside the subaperture. The good point would be that this WFS is running extremely fast on SPHERE $(1200 \mathrm{~Hz})$ and is delivering the WFS in parallel to the observing.

- Focal-plane wave-front sensing directly measures the impact of aberrations in the imaging focal plane. The DTTS delivers IR images continuously with the science observation. It is used to perform Tip-Tilt sensing but delivers actually more than this information. This WFS might be a good candidate, with a limited framerate of 1 second, and a limited field of view. 
Also, the DTTS is only used during IR observations (IRDIS or IFS instrument), but might be out of the field of view during the Visible observation (ZIMPOL instrument).

Masen Lamb from UVic worked as an invited researcher at LAM during 3 month, at the end of his $\mathrm{PhD}$. Following very first ideas and results explained in [1], he developed a complete phase diversity algorithm extension in OOMAO [2] able to use an aberration basis dedicated to LWE estimation. The results of his work are described in this conference proceeding [3].

Measuring the LWE from a focal-plane image directly points to phase diversity methods [4]. These methods are based on a model of image formation, accounting for phase and amplitude aberrations. Most of the time, the phase aberration is described on Zernike basis, or on a similar low-order basis (disc harmonics). Here we want to describe some phase aberrations that are badly defined by these basis, as they are containing high spatial frequencies. We have therefore defined a modified phase diversity which is essentially based on a modified basis. On top of the regular low order modes, we have added a dedicated 12 additional basis shown on Figure 6, containing piston tip and tilt polynomials defined on each of the 4 segments of the VLT pupil. These 12 additional modes are orthogonal one with respect to another (due to their completely distinct contribution in the pupil plane), but are not orthogonal to the usual Disc Harmonics basis. We are assuming anyway that the algorithm will be able to deal with this issue.
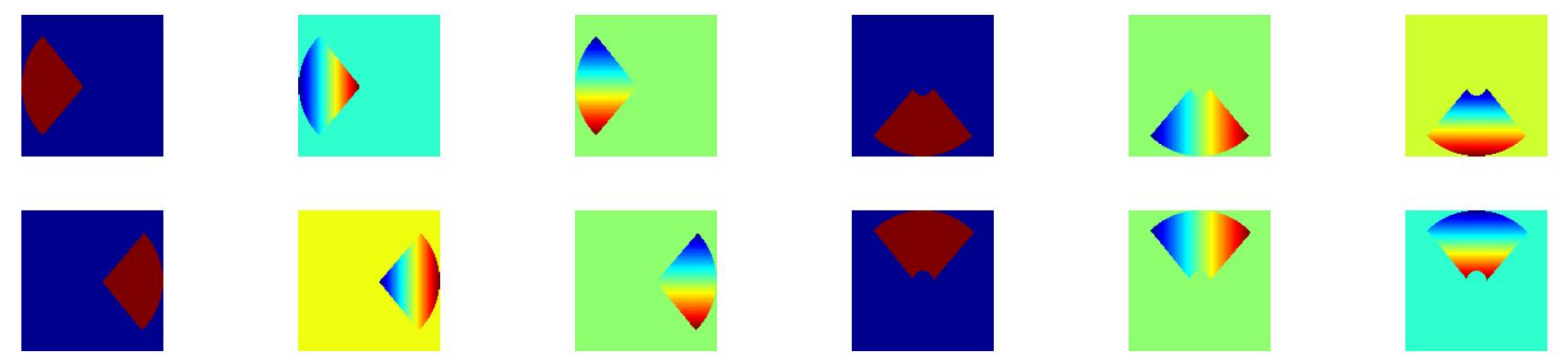

Figure 6: 12 additional modes used on top of 10 classical low order modes (disc harmonics) in order to measure the LWE pattern correctly from the focal plane.

The result of this Phase Diversity algorithm is extremely good, and shows a good capacity to measure the LWE both in high and low SNR regime. The Figure 7 shows a simulation result of this technique, showing how good the LWE has been estimated. The left side shows the PSF shape when corrugated with LWE. The level of LWE is $800 \mathrm{~nm}$ PV. The Strehl is very low: $47 \% \mathrm{H}$ band. The right side of Figure 7 shows the PSF simulated after the correction of estimated LWE. The correction assumes that the LWE pattern has been perfectly applied to the system. The Strehl ratio increases back to $96 \% \mathrm{H}$ band. Even if both the LWE and NCPA contributions have been estimated in this scheme, only the LWE contribution has been compensated for. The correcting NCPA on top of LWE would increase the image quality on DTTS, but not necessarily on the coronagraph. A more complete review of the technic performance is shown in detail in [3]. 


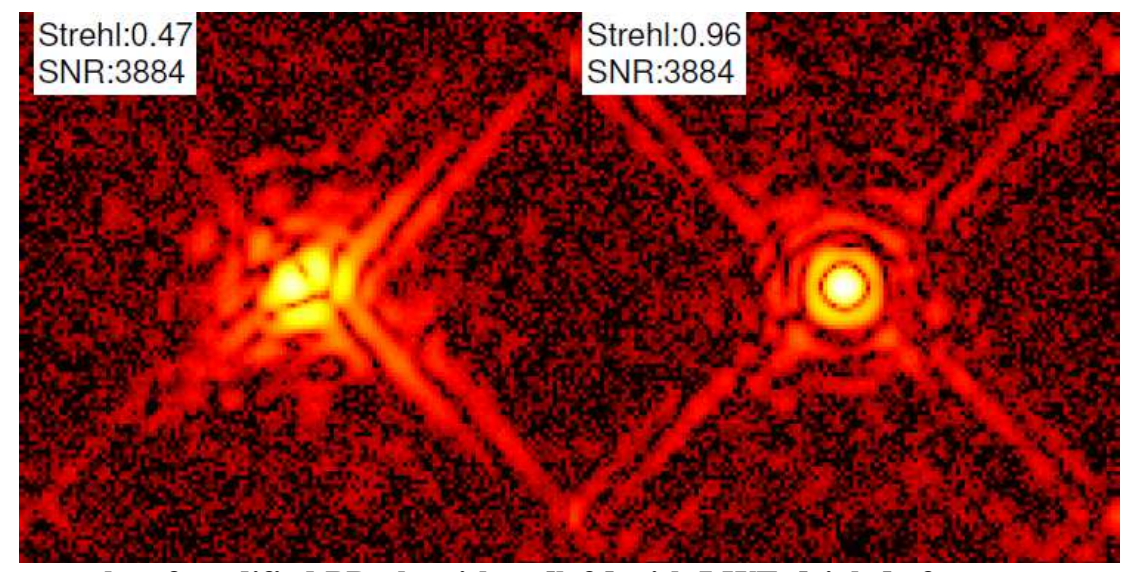

Figure 7: Simulation results of modified PD algorithm. [left] with LWE. [right] after measurement and perfect correction of $\mathbf{L W E}$.

\section{COMPENSATING THE LWE}

The first way to compensate for LWE pattern is of course to tackle down the root cause of the effect. This means modify the telescope in order to decrease the process that produces the LWE. The solutions are the following:

- Increase the wind speed at spider level

- Act on the spider temperature (with fluid circulation or else) in order to fit the air temperature

- Decrease the radiative transfer by choosing an alternate cover for the spider

- Design the spider profile to minimize the cross-section with the wind

The first is not recommended, because an increase of wind speed will end up in a reduced performance for AO. The second may introduce some additional turbulence, decreasing the performance of the system. The third is doable at VLT, but needs testing in order to choose the painting correctly for all spectral bandwidth of the instruments sharing the telescope. The fourth is doable with a telescope still under design

Introducing a static pattern with an XAO system is a process already used to compensate for the NCPA in AO system [5]. Such a procedure is based on a modification of the reference slopes of the WFS, which will drive the system to introduce the desired aberration when the loop is closed. Applying such a method with LWE is not an easy task, as the LWE pattern does not look like a classical low-order NCPA. LWE is the combination of high spatial frequencies (due to the sharp edges) but also low spatial frequencies (due to the tip-tilt defined on each of the quadrant).

In order to demonstrate the ability of SAXO to correct for the LWE pattern, we have driven a test on calibration source of SPHERE. This mode allows one to use the AO system on a point source, but without turbulence nor telescope environment. In this mode, the LWE pattern is of course not present. Our test consists in modifying the reference slope to see if the AO system is able to control it. 
Of course we have seen that the LWE does almost not interfere with the AO system. The mode we have tried to control is therefore a LWE pattern, filtered on the controlled modes of AO system. During this test, ZELDA has been used as a truth sensor.

We have generated a fake LWE pattern, with one quarter of the pupil shifted with a 100nm piston.

Figure 8 shows the ZELDA wave-front measurements at the coronagraph level. On left side, the LWE pattern has been applied directly on the HODM voltages. The actuators are shifted within a range of $2 \%$ of the HODM dynamic, and the pattern seen by ZELDA clearly shows the corresponding quarter also shifted. The typical size of the phase jump is of the order of a pitch $(2.5 \%$ of the pupil diameter), hence clearly less sharp than the original phase jump (less than $1 \%$ of pupil size). The direct projection on HODM voltage is made by the following operations

$$
\mathrm{v}=\mathrm{F}^{-1} \phi
$$

$v$ being the voltage to apply to the HODM, $F$ being the influence matrix (concatenation of influence functions phase), $F^{-1}$ is the inverse of $F . \phi$ is the phase to be projected. In this projection, all the possible degrees of freedom of the deformable mirror are used to fit the phase $\phi$.

On right side of Figure 8, the LWE pattern is applied via a modification of reference slopes, and a closed loop process. The modified slopes are computed as follows:

$$
\Delta \mathrm{s}=\mathrm{Cv}
$$

$\Delta s$ being the computed slopes modification, $C$ being the control matrix of the system, and $v$ being the previous voltages. The control matrix of the system is the result of the pseudo-inverse of the interaction matrix. It means that a certain number of modes has been filtered out by the inversion and that all degrees of freedom of the deformable mirror are not necessarily used to introduce the phase. The edge of the introduced defect is even less sharp than with the direct projection on HODM voltage.

This experiment is a clear demonstration that SPHERE is able to control the LWE pattern, at least on internal source.

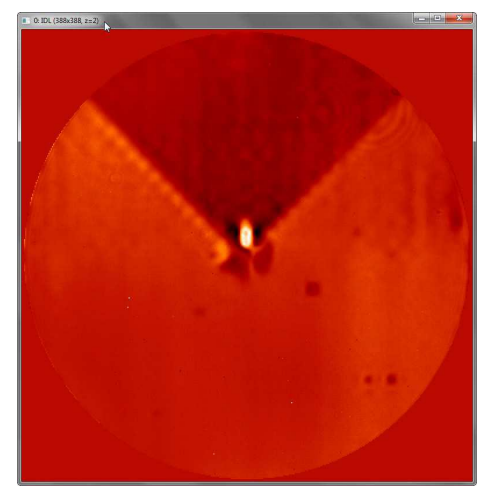

$$
\begin{gathered}
\text { Open-loop, } \\
\text { projection on HODM voltage }
\end{gathered}
$$

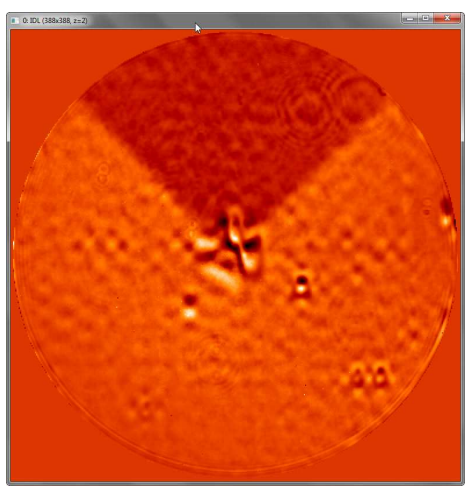

Closed-loop, Projection on reference slopes

Figure 8: ZELDA measurement of wave-front error, after introducing the LWE pattern in internal source. 


\section{CONCLUSION}

We have demonstrated in this paper a global and detailed comprehension of the LWE on SPHERE, at VLT observatory. The effect is a dome seeing effect, resulting from temperature difference between the spider and the incoming air flow. The scenario proposed in [1] has been demonstrated by mean of numerical simulations. Then the different steps of a LWE compensation method have been demonstrated. First we show that we are able to retrieve both the high and low spatial frequencies, thanks to a modified phase diversity algorithm. The usual modal basis for phase diversity has been increased by 12 additional modes describing the piston, tip and tilt aberrations restricted on each of the pupil quadrant. This method has been tested and validated thanks to numerical simulations, and shows very good results on both high and low SNR regimes. Lastly, the correction of the LWE with the SAXO instrument has been validated on an internal source, and shows that the correction of the LWE with a modification of reference slopes is doable. The perspectives of this work is to first to test the procedure on-sky with LWE present. The second step would be to modify the modal basis of the AO control law itself, in a similar way than the modified

phase diversity algorithm. This would imply to add the 12 piston-tip-tilt basis to the 990 controled Karunen-Loeve modes.

\section{REFERENCES}

[1] Low Wind Effect, the main limitation of the SPHERE instrument, AO4ELT4 conference, Lake Arrowhead 2015.

[2] Object-oriented Matlab adaptive optics toolbox R. Conan ; C. Correia Proc. SPIE 9148, Adaptive Optics Systems IV, 91486C (August 7, 2014); doi:10.1117/12.2054470

[3] Estimating Phase Errors from Pupil Discontinuities from simulated On Sky Data: Examples with VLT and Keck, M. Lamb et al (this conference)

[4] Phase Retrieval And Diversity In Adaptive Optics, R. A. Gonsalves, Opt. Eng. 21(5), 215829 (Oct 01, 1982). doi:10.1117/12.7972989

[5] Non-Common Path Aberrations measurement and pre-compensation, J.-F. Sauvage, JOSAA 2007 